\title{
Stage IVB Thyroid Gland Follicular Carcinoma AJCC v7
}

National Cancer Institute

\section{Source}

National Cancer Institute. Stage IVB Thyroid Gland Follicular Carcinoma A/CC v7. NCI

Thesaurus. Code C115036.

Stage IVB includes: T4b, Any N, M0. T4b: Very advanced disease. Tumor invades prevertebral fascia or encases carotid artery or mediastinal vessels. M0: No distant metastasis. (AJCC 7th ed.) 